International Journal of Bifurcation and Chaos, Vol. 17, No. 10 (2007) 3557-3563

(C) World Scientific Publishing Company

\title{
ENSEMBLE APPROACH FOR RECOVERING PHASE SYNCHRONIZATION FROM TIME SERIES
}

\author{
ISAO TOKUDA* and JÜRGEN KURTHS \\ Nonlinear Dynamics, Institute of Physics, \\ University of Potsdam, D-14415, Potsdam, Germany \\ *School of Information Science, \\ Japan Advanced Institute of Science and Technology, \\ Ishikawa 923-1292, Japan \\ *isao@jaist.ac.jp \\ ULRICH PARLITZ \\ Drittes Physikalisches Institut, Universität Göttingen, \\ Friedrich-Hund-Platz 1, D-37077, Göttingen, Germany
}

Received October 27, 2005; Revised February 5, 2006

\begin{abstract}
An ensemble approach is presented for the reconstruction of a phase synchronization diagram from time series. As an example system, we analyze a forced Colpitts oscillator to show that synchronization diagram reconstructed by a single nonlinear model depends sensitively upon the model parameters, which should be estimated with a considerable amount of care. This dependence can be crucial for a precise recovery of the synchronization phenomena. To overcome this weakness, an ensemble approach is introduced. Two types of techniques, namely, (I) ensemble regression and (II) ensemble classification, are developed to show that they provide much more robust and reliable reconstruction of the synchronization diagram compared to the conventional single modeling approach.
\end{abstract}

Keywords: Phase synchronization; nonlinear modeling; ensemble technique; chaos.

\section{Introduction}

Synchronization is a fundamental phenomenon of coupled or forced nonlinear oscillators, which are common in nature and engineering. Up to date, four basic types of chaotic synchronization, namely, complete synchronization [Fujisaka \& Yamada, 1983; Pecora \& Carroll, 1990], generalized synchronization [Rulkov et al., 1995; Kocarev \& Parlitz, 1996; Josic, 1998], phase synchronization [Rosenblum et al., 1996], and lag synchronization [Rosenblum et al., 1997], have been found. Phase synchronization (PS) of coupled or periodically forced nonlinear systems has found many applications both in laboratory experiments and natural systems [Kurths, 2000; Pikovsky et al., 2001;
Boccaletti et al., 2002]. The next important step is to analyze the synchronization phenomena from time series data observed from such experimental or natural systems. So far, several techniques have been developed to detect PS in the underlying coupled nonlinear systems from bivariate or multivariate data [Kurths, 2000; Pikovsky et al., 2001; Rosenblum et al., 2002; Osipov et al., 2003; Romano et al., 2005]. Although such techniques have been shown to be quite efficient even for noisy and nonstationary data, the problem of modeling the synchronization phenomena from data remains open. By using such models, it is of special interest to infer a synchronization diagram, which yields the regimes of PS, non-PS, and borderlines between 
both, depending upon the system parameters such as the coupling strength and the frequency mismatch between interacting nonlinear oscillators. By recovering such synchronization diagram from only a few sets of experimental data, a deeper insight into the underlying coupled systems can be gained. This problem formulation is quite practical for situations in which an extensive synchronization experiment is not possible and only limited data sets can be experimentally recorded. To retrieve the synchronization regime, construction of a nonlinear model, which is parameterized by forcing conditions, such as the coupling strength and the forcing frequency, from the recorded data is essential. In a recent study, we have introduced a novel technique for constructing such a parameterized family of nonlinear models based on an artificial neural network and its parameter reduction by the singular value decomposition [Tokuda et al., 2001]. Our technique has been successfully applied to prototypical PS models, asymmetric vocal fold model [Tokuda \& Herzel, 2005], and also to experimental data from a paced plasma discharge tube [Tokuda et al., 2001] and a chaotic $\mathrm{CO}_{2}$ laser [Tokuda et al., 2004]. For practical application of our approach to a variety of real experimental systems, it is important to note that this technique has a strong dependence upon the model selection, especially on the parameter estimation of the nonlinear models. The approach is not very reliable because the result is so sensitive to the estimated model parameters. The main purpose of the present letter is to introduce the idea of ensemble technique [Hansen \& Salamon, 1990; Meir, 1995; Krogh \& Sollich, 1997; Naftaly et al., 1997] in order to realize a robust reconstruction of the synchronization diagrams from time series data.

\section{Modeling Technique}

First, we state our problem. Suppose we have a forced nonlinear oscillator whose dynamical state and forcing signal are simultaneously recorded into bivariate time series $\{x(t), y(t)\}$. As the forcing signal, sinusoidal forcing $y(t)=E \sin (\nu t)$ is considered. Among several dynamical components of the nonlinear oscillator, only a single variable $x(t)$ is observed. For $M$ sets of different forcing conditions $\left\{\nu_{i}, E_{i}\right\}_{i=1, \ldots, M}$, the bivariate data are recorded. Based on the $M$ sets of the bivariate data $\{x(t), y(t)\}$, our task is to predict for which parameters of the forcing frequency $\nu$ and amplitude $E$ the forced system is in the regime of PS.
This condition is practical for experimental situations, under which an extensive exploration of the synchronization property is not possible. For instance, in neuroscience, a response characteristic of a single neuron to sinusoidal forcing provides an important clue. Due to its limited life-time, however, it is almost impossible to investigate the response property of the physiological neuron to every combination of the forcing frequency and the amplitude. It is therefore an awaited technique to estimate the synchronization diagram from only few sets of recorded data.

Our modeling technique is as follows. We embed the bivariate time series $\{x(t), y(t)\}$ into delay coordinates $X(t)=\{x(t), x(t-\tau), \ldots, x(t-(d-1) \tau)\}$, $Y(t)=\{y(t), y(t-\tau), \ldots, y(t-(d-1) \tau)\}(d:$ embedding dimension, $\tau$ : time lag) and suppose according to the embedding theorem [Takens, 1981; Sauer et al., 1991] that there exists the following underlying dynamics

$$
\frac{d X}{d t}=F(X(t), Y(t))
$$

The main point of our modeling is to construct a nonlinear function $\tilde{F}$, that approximates Eq. (1). If the original forced dynamics is precisely modeled, the regime of PS as well as non-PS in the parameter space of the forcing frequency $\nu$ and amplitude $E$ can be predicted by studying the model $\tilde{F}$ instead of the original forced system. The modeling procedure is summarized as follows.

(P1) The embedding dimension $d$ and the time lag $\tau$ are determined. The embedding dimension can be chosen via conventional techniques such as the false nearest neighbor technique [Abarbanel, 1996]. To determine the time lag, the first zero-crossing point of the autocorrelation function is exploited.

(P2) By Euler's discretization, the dynamical equation (1) is approximated as

$$
X(t+1)=X(t)+\Delta t \cdot \tilde{F}(X(t), Y(t)),
$$

where $\Delta t$ stands for a sampling interval. Although it is a delicate procedure to discretize continuoustime nonlinear dynamics by the Euler's formula, it has been reported earlier that the Euler's scheme with a relatively large sampling $\Delta t$ is capable of approximating the global structure of chaotic dynamics including bifurcations [Tokuda et al., 1996]. Then, the nonlinear model $\tilde{F}(X, Y)$ is realized by a three-layer feed-forward neural network [Rumelhart et al., 1986], having 2d-units in the input layer, $d$-units in the output layer, and $h$-units 
in the middle layer. To fit the nonlinear model to the data, the model parameters $\Omega$ are optimized with respect to the cost function

$$
\begin{aligned}
E_{D}(\Omega)= & \sum_{E, \nu} \sum_{t=(d-1) \tau}^{N_{D}-K} \sum_{k=1}^{K}\{\tilde{X}(t+k, E, \nu) \\
& -X(t+k, E, \nu)\}^{2}
\end{aligned}
$$

where the summation $\sum_{E, \nu}$ applies to all data $\{x(t), y(t)\}\left(t=1,2, \ldots, N_{D}\right)$ recorded with different forcing conditions $\left\{\nu_{i}, E_{i}\right\}_{i=1, \ldots, M}, N_{D}$ stands for the number of the data points, and $\tilde{X}(t+k)$ corresponds to $k$ th iterated state of the dynamics $\tilde{X}(t+k)=\tilde{X}(t+k-1)+\Delta t \cdot \tilde{F}(\tilde{X}(t+k-1), Y(t))$ started from $\tilde{X}(t)=X(t)$. The parameter optimization is carried out by minimizing the cost function $E_{D}$ by the quasi-Newton method [Luenberger, 1973].

(P3) By free-running of the nonlinear model (2), which is forced by the sinusoidal term $Y(t)=$ $\{E \sin (\nu t), E \sin (\nu t-\tau), \ldots, E \sin (\nu t-(d-1) \tau)\}$, the frequency $\tilde{\nu}$ of the forced model is computed. A unit phase of $2 \pi$ is defined as a duration between one local minimum of the first component of $X$ and the following one. By counting the number $N_{m}$ of the local minima during a period $T_{f}$, the frequency of the free-running model (2) can be computed as $\tilde{\nu}=2 \pi N_{m} / T_{f}$. The relative frequency difference between the model and the forcing is then given as $\Delta \nu=(\tilde{\nu}-\nu) / \nu$. By changing the forcing amplitude $E$ and frequency $\nu$ of the forcing term in (2), synchronization diagram, which shows dependence of the frequency difference $\Delta \nu$ on the forcing condition $\{E, \nu\}$, is drawn.

The key point of the present technique lies on the generalization capability of the nonlinear models. Namely, the model should provide good approximations not only on the recorded conditions $\left\{\nu_{i}, E_{i}\right\}_{i=1, \ldots, M}$ but further on unknown conditions, which have not yet been experimented. The well known problem for this modeling is the over-fitting, where the model fits too closely to the recorded data and predicts poorly on unknown data points. Hence, optimization of the nonlinear models requires a delicate procedure. To deal with this problem, we introduce an ensemble averaging technique [Hansen \& Salamon, 1990; Meir, 1995; Krogh \& Sollich, 1997; Naftaly et al., 1997]. The basic idea of the ensemble averaging is to construct many different nonlinear models from the data and to utilize all the models as an ensemble in order for the prediction
[Langer \& Parlitz, 2004]. In our approach, such elements of nonlinear models are collected by using neural networks $\tilde{F}\left(\Omega_{i}\right)(i=1, \ldots, Q)$ having the same architecture but having different parameter values $\Omega_{i}$, which are obtained by the optimization procedure of $(\mathrm{P} 2)$ started from different random initial condition. For the reconstruction of the synchronization diagram, $Q$ sets of nonlinear models are incorporated in the following two different ways, namely, (I) ensemble regression (ER) and (II) ensemble classification (EC).

(I) In the ER approach, the ensemble model $G$ is constructed by averaging $Q$ sets of nonlinear models as

$$
G(X(t), Y(t))=\frac{1}{Q} \sum_{i=1}^{Q} \tilde{F}\left(\Omega_{i}, X(t), Y(t)\right) .
$$

In the procedure (P3), the ensemble model $G$ is utilized for the free-running as $X(t+1)=$ $X(t)+\Delta t \cdot G(X(t), Y(t))$ to predict the regime of synchronization.

(II) In the EC approach, each nonlinear model $\tilde{F}\left(\Omega_{i}\right)$ is simulated to make a prediction on whether a forcing condition $(E, \nu)$ gives rise to synchronization or not. If majority of the models "vote" for synchronization, the condition $(E, \nu)$ is determined as a synchronized condition and otherwise as a nonsynchronized condition.

We note that the present ensembling procedure increases the computational cost. Compared with the conventional approach based on a single nonlinear model, the ensemble approach, which utilizes $Q$ nonlinear models, roughly requires $Q$ times more computations. Despite this computational disadvantage, it is worthwhile developing a more reliable technique to reconstruct the synchronization diagram.

\section{Application to the Forced Colpitts Oscillator}

Let us apply our technique to a periodically forced version of the Colpitts oscillator [Kennedy, 1994]:

$$
\begin{aligned}
C_{1} \frac{d V_{C_{1}}}{d t} & =I_{L}-\beta g\left(V_{C_{2}}\right), \\
C_{2} \frac{d V_{C_{2}}}{d t} & =-\frac{V_{E}+V_{C_{2}}+E \cos \nu t}{R}-I_{L}-g\left(V_{C_{2}}\right), \\
L \frac{d I_{L}}{d t} & =V_{C}-V_{C_{1}}+V_{C_{2}}-R_{L} I_{L},
\end{aligned}
$$


where $g(V)=\left(I_{S} / \beta\right) \exp \left(V / V_{T}\right)$. Realistic circuit condition is simulated by the fourth order RungeKutta method with an integration step of $\pi / 8192$ using the following parameter values: $C_{1}=4.7 \mu \mathrm{F}$, $C_{2}=4.7 \mu \mathrm{F}, R=0.39 \mathrm{k} \Omega, L=0.0027 \mathrm{H}, R_{L}=$ $0.025 \mathrm{k} \Omega, \beta=200, V_{T}=0.026 \mathrm{~V}, I_{S}=1.4$. $10^{-10} \mathrm{~mA}, V_{C}=3.3 \mathrm{~V}, V_{E}=-3.3 \mathrm{~V}$. Figure 1 shows chaotic attractor from the Colpitts oscillator in the $\left(V_{C_{2}}, I_{L}\right)$ space. Around $\left(V_{C_{2}}, I_{L}\right) \approx(0.5$, $7)$, a single rotation center is discernible. Such a property associated with a single center is termed phase-coherence, which provides a unique phase for chaotic attractor independent of the phase definition [Pikovsky et al., 2001]. Due to this phasecoherent property, the phase can be well defined for the Colpitts oscillator. Among various definitions proposed as a phase of chaotic attractor, this study defines a unit phase of $2 \pi$ as a duration between a local minimum of the third component $I_{L}$ and the following one. By numerical simulation of the forced equations, we have clearly observed a chaotic PS, giving rise to a well-pronounced Arnold tongue in a region of relatively weak forcing strength.

For three sets of different forcing conditions $\{\nu, E\}=\{0,0\},\{12.02,0.12\},\{12.63,0.12\}$, the corresponding bivariate time series $\left\{I_{L}(t), E \cos \nu t\right\}$ were recorded from the Colpitts oscillator and its forcing. As indicated by crosses in Fig. 2(a), the data sets were collected from the nonsynchronized chaotic regime, since this regime provides a rich data for retrieving the synchronization structure [Tokuda et al., 2001]. The sampling interval was set to be $\Delta t=\pi / 256$, whereas the total recording interval was set as $1200 \Delta t$ (i.e. $N_{D}=1200$ ). In the

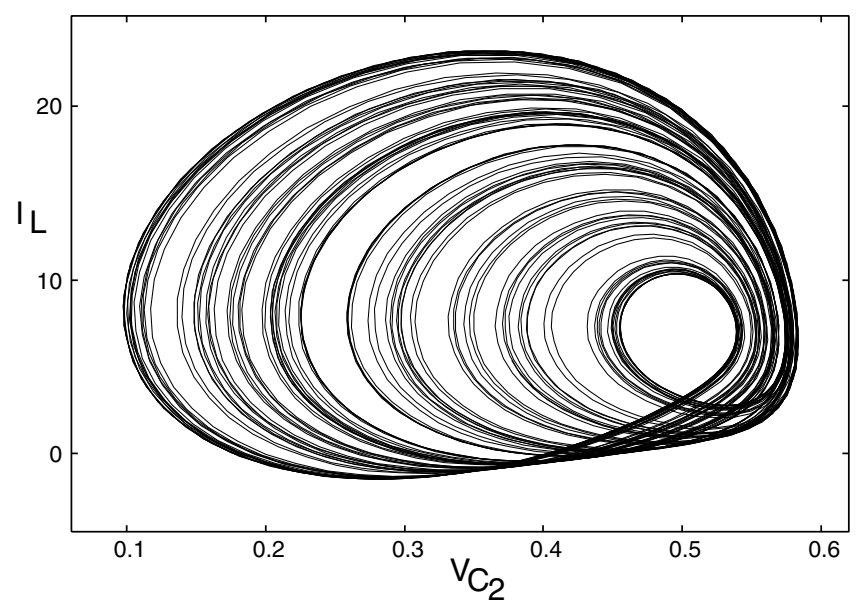

Fig. 1. Chaotic attractor from the Colpitts oscillator in the $\left(V_{C_{2}}, I_{L}\right)$ space. modeling process of $(\mathrm{P} 1)$, the embedding dimension and the time lag were chosen as $(d, \tau)=(3,11 \Delta t)$. In (P2), number of units in the middle layer of the neural network was determined as $h=14$ by the cross-validation technique. With respect to 400 sets of different initial conditions uniformly distributed over $[-0.5,0.5], 400$ sets of model parameters $\left\{\Omega_{i}: i=1,2, \ldots, 400\right\}$ were obtained. The maximum iteration number for the quasi-Newton method was set to be $10^{4}$, whereas the integration interval of the cost function (3) was set to be $K=32$. Among 400 sets of the obtained model parameters $\left\{\Omega_{i}: i=1,2, \ldots, 400\right\}, 285$ sets, which give rise to a natural frequency, associated with self-sustained oscillations of the model (2) without forcing, ranging between $12.02<\tilde{\nu}<12.630$, were extracted and they were randomly selected to construct the ensemble model. The random selection was carried out in a way that a same single model was not selected twice as elements of one ensemble model.

Figure 2(a) shows the synchronization diagrams of the forced Colpitts oscillator and ten sets of the nonlinear models without utilizing the ensemble technique $(Q=1)$. Abscissa and ordinate respectively indicate the forcing frequency and amplitude, varied as $(\nu, E) \in[12: 12.65] \times[0: 0.18]$. Inside and outside of the borderline correspond to the regimes of PS and non-PS, respectively. The condition for PS is defined as $|\Delta \nu|<0.002$. Although the borderlines of the ten models tend to reproduce similar configuration to the original borderline, some model borderlines are deviated largely from the true one. This indicates that reconstruction of the PS diagrams from data sensitively depends upon the model parameters, which should be estimated carefully. Moreover, estimation of the borderline based upon a single model is not highly reliable due to its large confidence interval. Figure 2(b), on the other hand, shows synchronization diagrams of the original and ten sets of ensemble classification models with $Q=17$. The difference in the ten sets is due to different selection of the ensemble elements from 285 single models $\tilde{F}\left(\Omega_{i}\right)$. The borderlines of the ten ensemble models reproduce the basic configuration of the original borderline almost precisely. Compared with Fig. 2(a), the variation among the nonlinear models are much smaller, giving rise to a highly reliable and robust reconstruction of the PS diagram. We note that the estimated borderline is less precise on the right-hand side than on the left-hand side. The cause of this right-left 


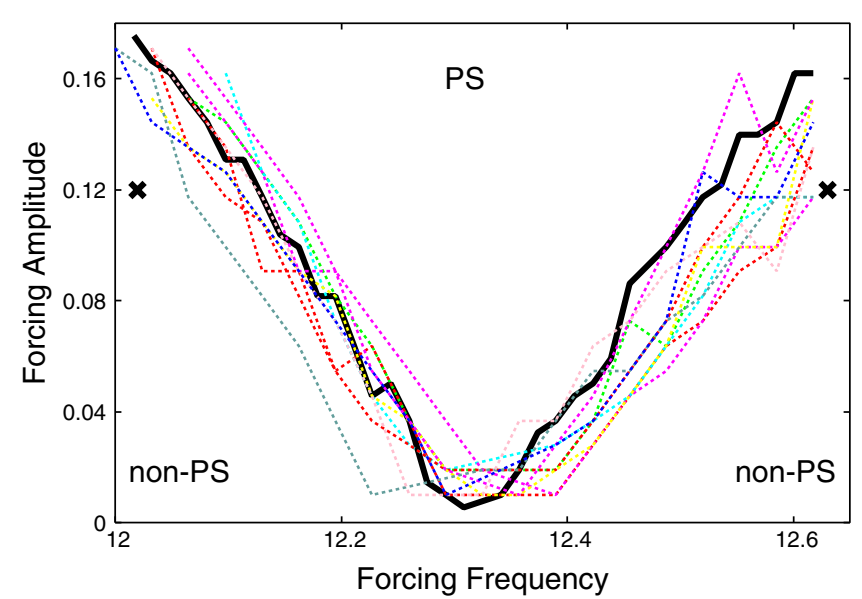

(a)

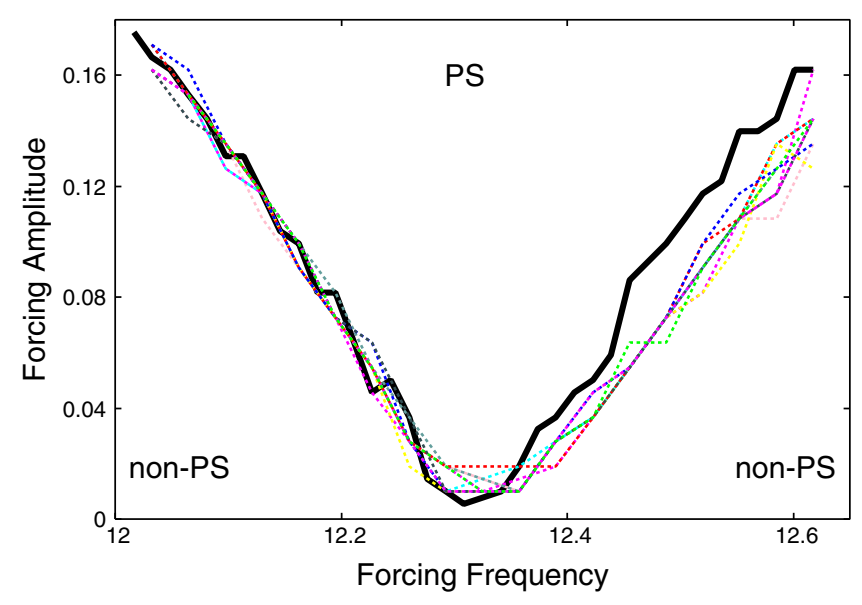

(b)

Fig. 2. Synchronization diagrams of the original forced Colpitts system (black bold line in both (a) and (b)), ten different single nonlinear models (dotted lines in different colors in (a)), and ten different realizations of the ensemble classification technique with $Q=17$ (dotted lines in different colors in (b)). The cross points in (a) correspond to the forcing conditions utilized for the data modeling. Inside and outside of the borderline correspond to the regime of PS and non-PS, respectively. The forcing frequency and the amplitude are varied as $(\nu, E) \in[12: 12.65] \times[0: 0.18]$.

unbalance, which seems to be due to an inherent property of the underlying Colpitts system, is at this point not well understood.

To quantify the similarity between the original and the model PS diagrams, a normalized mean squared error (NMSE) was computed as follows. On $N \times N$ grid points uniformly distributed over the parameter space $(\nu, E) \in[12: 12.65] \times[0: 0.18]$, frequency difference $\Delta \nu\left(\nu_{i}, E_{j}\right)$ are computed for $i, j=1, \ldots, N$. If the point $(i, j)$ is in a PS regime, that is, $\left|\Delta \nu\left(\nu_{i}, E_{j}\right)\right|<0.002$, we denote its state

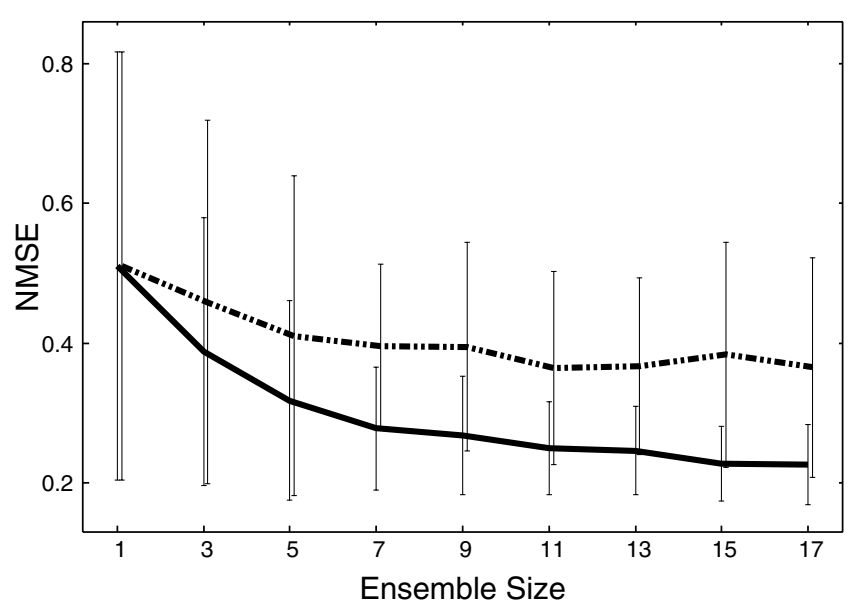

(a)

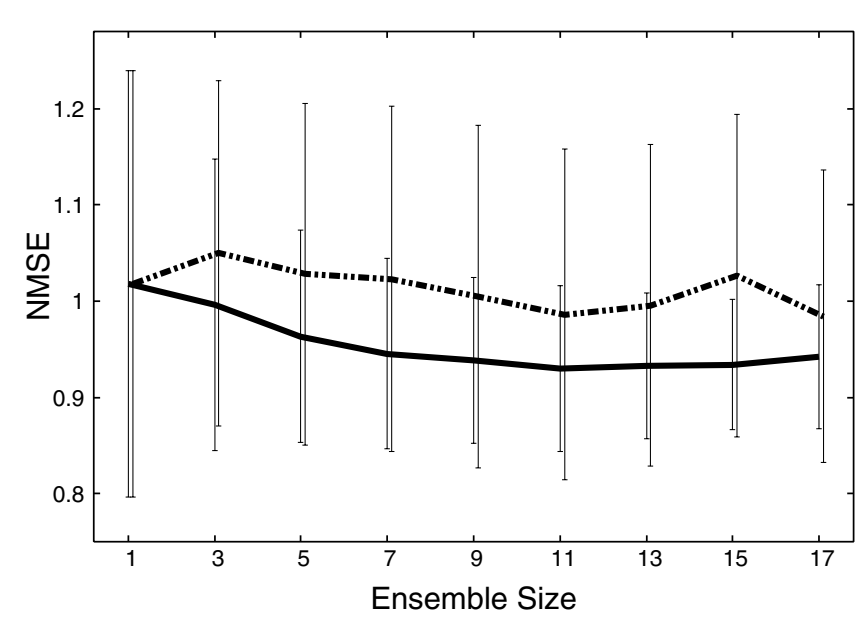

(b)

Fig. 3. Dependence of the normalized mean squared error (NMSE) on the ensemble size $Q$. The solid line corresponds to the ensemble classification, whereas the dotted line corresponds to the ensemble regression. The NMSE was averaged over 100 different realizations of the ensemble method. The error bars correspond to the standard deviation. (a) and (b) correspond to the case of utilizing the original time series and the surrogate data, respectively, for the modeling.

by $\eta(i, j)=1$. Otherwise, the state is denoted by $\eta(i, j)=-1$. The NMSE of the model $\left\{\eta_{m}(i, j)\right\}$ against the original $\left\{\eta_{o}(i, j)\right\}$ is then computed by $E=\sum_{i, j}\left\{\eta_{m}(i, j)-\eta_{o}(i, j)\right\}^{2} / \sum_{i, j}\left\{\eta_{o}(i, j)-\bar{\eta}_{o}\right\}^{2}$, where $\bar{\eta}_{o}=\left(1 / N^{2}\right) \sum_{i, j} \eta_{o}$. Figure $3($ a) shows the dependence of the NMSE $(N=20)$ on the size of the ensemble $Q$. The NMSE was averaged over 100 different realizations of the model selection, whereas the error-bars indicate the standard deviation. For both ER and EC curves, we see that, as the ensemble size $Q$ is increased, the NMSE decreases 
monotonically, realizing an improved reconstruction of the synchronization diagram. An increase in the ensemble size results in a smaller error-bar, which implies a robust estimation of the synchronization diagram with smaller confidence intervals. We also note that smaller errors for the EC curve indicate that the EC is more effective than the ER. Our preliminary experiments, however, suggest that the relationship between EC and ER strongly depends upon the quality of individual elements of the single nonlinear models. The ER provides a good improvement when the individual nonlinear models are rather poor. Further investigation is necessary to obtain more conclusive results.

Finally, we present a surrogate data analysis [Theiler et al., 1992] to examine our modeling technique. In this analysis, dynamical correlation between two components of the data $\left\{I_{L}(t)\right.$, $E \cos \nu t\}$ used for our modeling is destroyed as follows. First, dynamical component $I_{L}(t)$ from the Colpitts oscillator remains unchanged. Then, a shift change in time is made for the forcing signal as $E \cos \nu(t+\delta t)$, where $\delta t$ stands for the shift constant. The obtained surrogate data $\left\{I_{L}(t), E \cos \nu(t+\delta t)\right\}$ shuffles dynamical correlation between the two components.

From two sets of the bivariate time series $\left\{I_{L}(t), E \cos \nu t\right\}$ forced respectively with conditions $\{\nu, E\}=\{12.02,0.12\},\{12.63,0.12\}$, their corresponding surrogate data $\left\{I_{L}(t), E \cos \nu(t+\delta t)\right\}$ were created. It is essential that the shift constant was set differently as $\delta t=15 \Delta t, 30 \Delta t$ for the two data sets. Together with the nonforced data $\left\{I_{L}(t), 0\right\}$, the surrogate data were exploited for the ensemble reconstruction of the PS diagram. The modeling conditions were set exactly the same as the previous ones. Figure 3(b) shows dependence of the NMSE on the ensemble size $Q$. In contrast to the case that no surrogate data were utilized [Fig. 3(a)], large NMSE values close to $E \approx 1$, indicating unreliable reconstruction of the PS diagram, have been obtained. With an increase in the ensemble size $Q$, no significant decrease in the error is observed for both ER and EC curves. This implies that the dynamical correlation between the bivariate data is essential for the reconstruction of the PS diagram. The present ensemble technique detects such a correlation highly sensitively to recover synchronization structure of the underlying forced nonlinear system.

\section{Conclusions}

In conclusion, an ensemble technique has been introduced for the reconstruction of the synchronization diagrams from time series. With an application to simulated data from the forced Colpitts oscillator, we have shown that the ensemble technique provides much more robust and reliable results compared to the conventional single modeling technique. Our future studies will focus on (1) effect of observational and dynamical noise on the ensemble technique, (2) combinatory use of both ER and EC techniques, (3) extension of the present approach to nonphase-coherent system such as the funnel-type Rössler oscillator by adopting a recent technique of detecting phase in a nonphase-coherent system [Romano et al., 2005], and (4) application of this approach to experimental data from real electronic circuits.

\section{Acknowledgments}

I. Tokuda was supported by the Alexander-vonHumboldt Foundation for the research fellowship. J. Kurths acknowledges support from the EU-Network of Excellence BIOSIM (Contract No 005137).

\section{References}

Abarbanel, H. D. I. [1996] Analysis of Observed Chaotic Data (Springer-Verlag, NY).

Boccaletti, S., Kurths, J., Osipov, G., Valladares, D. L. \& Zhou, C. [2002] "The synchronization of chaotic systems," Phys. Rep. 366, 1-101.

Fujisaka, H. \& Yamada, T. [1983] "Stability theory of synchronized motion in coupled-oscillator systems," Prog. Theor. Phys. 69, 32-47.

Hansen, L. \& Salamon, P. "Neural network ensembles," [1990] IEEE Trans. PAMI 12, 993-1001.

Josic, K. [1998] "Invariant manifolds and synchronization of coupled dynamical systems," Phys. Rev. Lett. 80, 3053-3056.

Kennedy, M. P. [1994] "Chaos in the Colpitts oscillator," IEEE Trans. Circuits Syst. 41, 771-774.

Kocarev, L. \& Parlitz, U. [1996] "Generalized synchronization, predictability, and equivalence of unidirectionally coupled dynamical systems," Phys. Rev. Lett. 76, 1816-1819.

Krogh, A. \& Sollich, P. [1997] "Statistical mechanics of ensemble learning," Phys. Rev. E 55, 811-825.

Kurths, J. (ed.) [2000] "Phase synchronization and its applications," Special Issue of Int. J. Bifurcation and Chaos 10(11). 
Langer, G. \& Parlitz, U. [2004] "Modeling parameter dependence from time series," Phys. Rev. E 70, 056217.

Luenberger, D. G. [1973] Linear and Nonlinear Programming (Addison-Wesley).

Meir, R. [1995] "Bias, variance and combination of least squares estimators," in Advances in Neural Information Processing Systems, eds. Tesauro, G., Touretzky, D. \& Leen, T., Vol. 7 (MIT Press), pp. 295.

Naftaly, U. Intrator, N. \& Horn, D. [1997] "Optimal ensemble averaging of neural networks," Network: Comput. Neural Syst. 8, 283-296.

Osipov, G., Hu, B., Zhou, C., Ivanchenko, M. \& Kurths, J. [2003] "Three types of transitions to phase synchronization in chaotic oscillators," Phys. Rev. Lett. 91, 024101.

Pecora, L. M. \& Carroll, T. L. [1990] "Synchronization in chaotic systems," Phys. Rev. Lett. 64, 812-824.

Pikovsky, A. Rosenblum, M. \& Kurths, J. [2001] Synchronization - A Universal Concept in Nonlinear Sciences (Cambridge University Press, Cambridge).

Romano, M. C., Thiel, M., Kurths, J., Kiss, I. Z. \& Hudson, J. [2005] "Detection of synchronization for nonphase coherent and non-stationary data," Europhys. Lett. 71, 466-472.

Rosenblum, M. G., Pikovsky, A. S. \& Kurths, J. [1996] "Phase synchronization of chaotic oscillators," Phys. Rev. Lett. 76, 1804-1807.

Rosenblum, M. G., Pikovsky, A. S. \& Kurths, J. [1997] "From phase to lag synchronization in coupled chaotic oscillator," Phys. Rev. Lett. 78, 4193-4196.

Rosenblum, M. G., Cimponeriu, L., Bezerianos, A., Patzak, A. \& Mrowka, R. [2002] "Identification of coupling direction: Application to cardiorespiratory interaction," Phys. Rev. E 65, 041909.

Rulkov, N. F., Sushchik, M. M., Tsimring, L. S. \& Abarbanel, H. D. I. [1995] "Generalized synchronization of chaos in directionally coupled chaotic systems," Phys. Rev. E 51, 980-994.

Rumelhart, D. E. McClelland, J. L. \& the PDP Research Group [1986] Parallel Distributed Processing (MIT Press, Cambridge).

Sauer, T., York, J. A. \& Casdagli, M. [1991] "Embedology," J. Stat. Phys. 65, 579-616.

Takens, F. [1981] "Detecting strange attractors in turbulence," Dynamical Systems and Turbulence, Lecture Notes in Mathematics, Vol. 898, eds. Range D. A. \& Young, L. S. (Springer, Berlin), pp. 366-381.

Theiler, J., Eubank, S., Longtin, A., Galdrikian, B. \& Farmer, J. D. [1992] "Testing for nonlinearity in time series: The method of surrogate data," Physica D 58, 77-94.

Tokuda, I., Kajiwara, S., Tokunaga, R. \& Matsumoto, T. [1996] "Recognizing chaotic time-waveforms in terms of a parametrized family of nonlinear predictors," Physica D 95, 380-395.

Tokuda, I., Kurths, J. \& Rosa, Jr. E. [2001] "Learning phase synchronization from non-synchronized chaotic regimes," Phys. Rev. Lett. 88, 014101.

Tokuda, I., Kurths, J., Allaria, E., Meucci, R., Boccaletti, S. \& Arecchi, F. T. [2004] "Predicting phase synchronization in a spiking chaotic $\mathrm{CO}_{2}$ laser," Phys. Rev. E 70, 035204R.

Tokuda, I. \& Herzel, H. [2005] "Detecting synchronizations in an asymmetric vocal fold model from time series data," Chaos 15, 013702. 